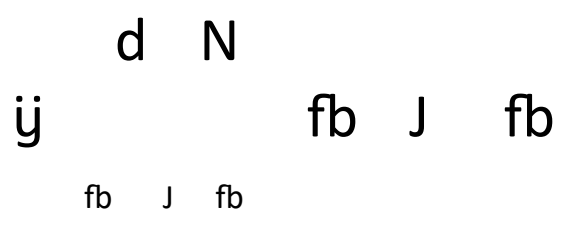

$\mathrm{fb} J \mathrm{fb}$

www.gjsoc.eu

\title{
Modeling Azerbaijan's action process concerning Nagorno-Karabakh and the occupied territories
}

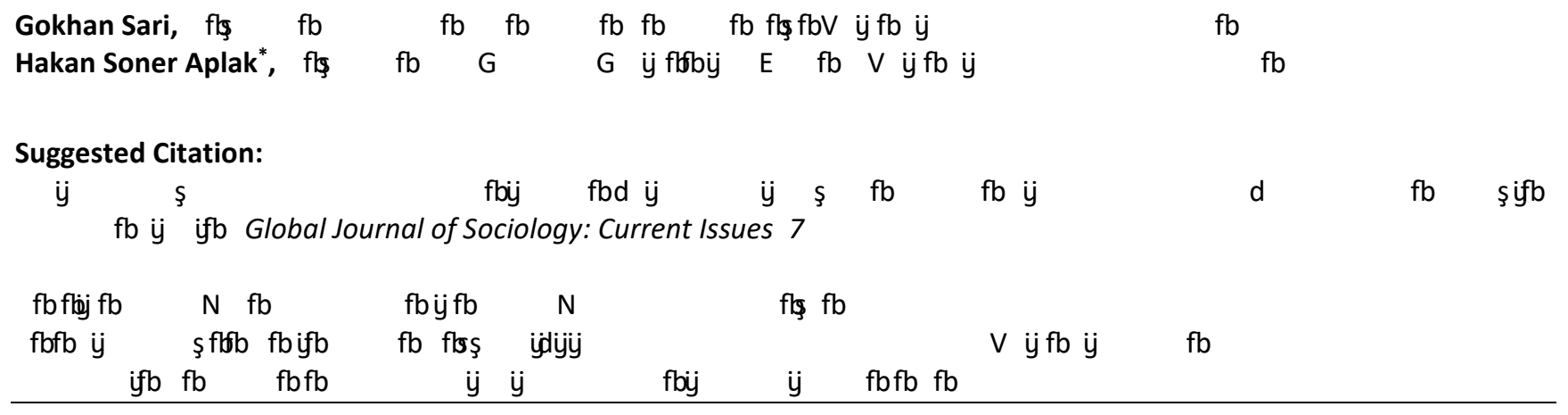

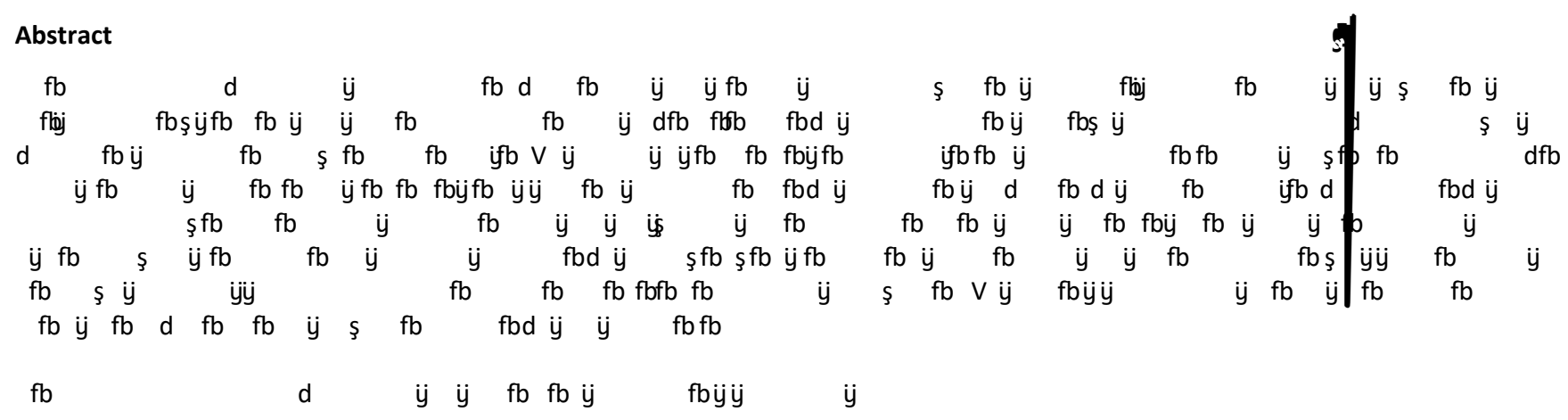




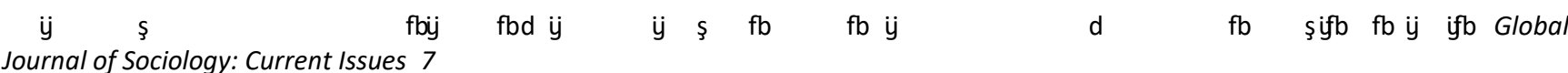

1. Introduction

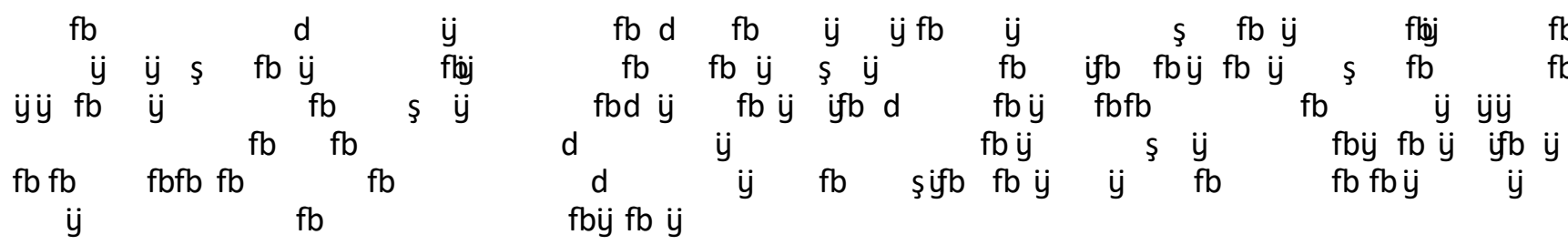




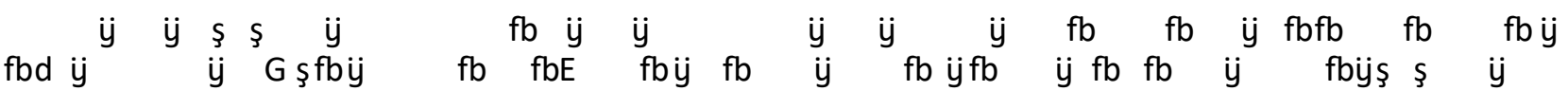

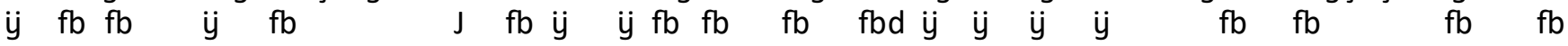

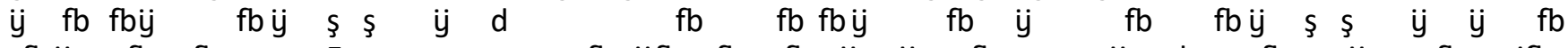

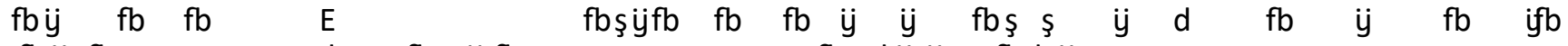
$\mathrm{fb} \ddot{\mathrm{j}} \mathrm{fb} \quad \mathrm{d} \quad \mathrm{fb} \quad \mathrm{y} \mathrm{fb} \quad \mathrm{flog} d \mathrm{~d} \dot{\mathrm{y}} \mathrm{ij} \quad \mathrm{fbd} \ddot{\mathrm{y}}$

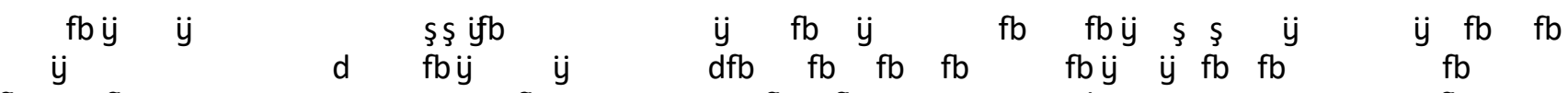

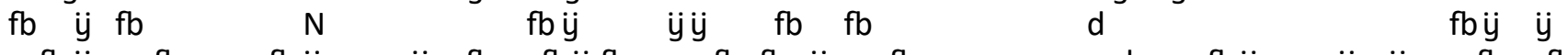

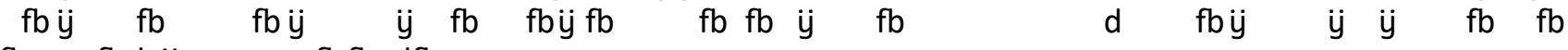
$\mathrm{fb} \quad \mathrm{fbd} \dot{\mathrm{j}} \quad \mathrm{fb} f \mathrm{dfb}$

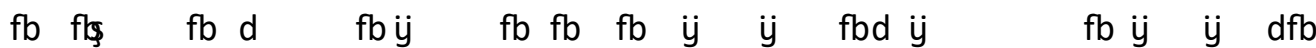

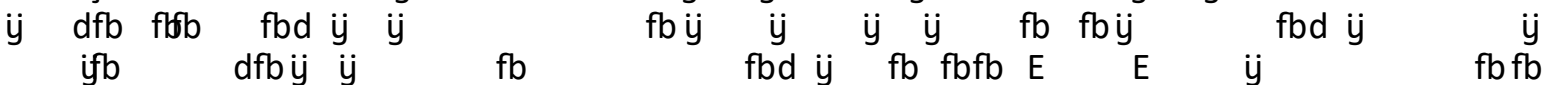

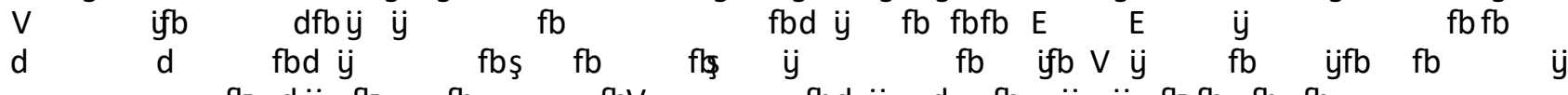 flos $d \ddot{y}$ flos fb fbV fbd $\ddot{y} \quad d \quad f b$ ij $\ddot{y}$ flog fb fb fb

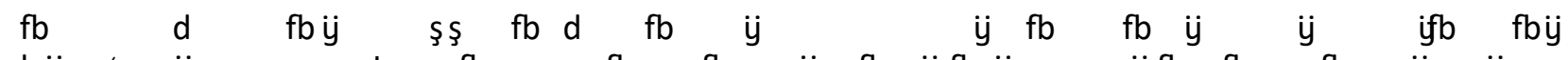

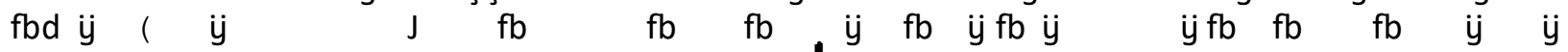

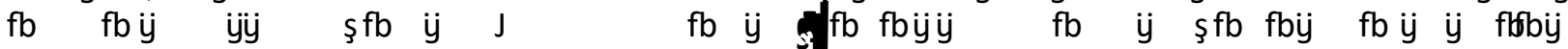

$$
\begin{aligned}
& \text { ij } \stackrel{\mathrm{fb}}{\mathrm{y}} \\
& \text { d } G \quad s ̧ f b \quad f b \text { ij }
\end{aligned}
$$

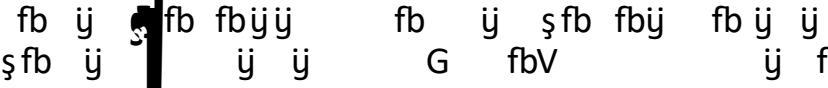

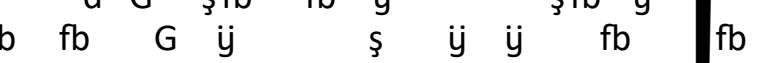

$$
\begin{aligned}
& \mathrm{fb} f b \quad \mathrm{~s} f \mathrm{fb} \text { fb } \mathrm{j} f \mathrm{fb} \dot{\mathrm{y}}
\end{aligned}
$$

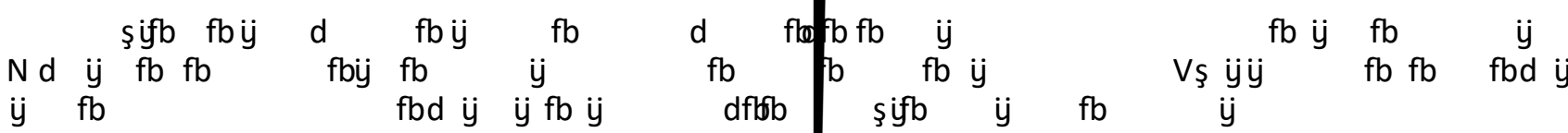

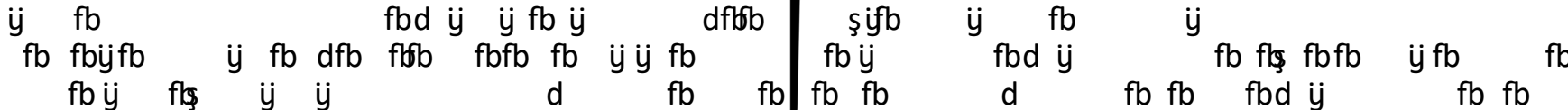

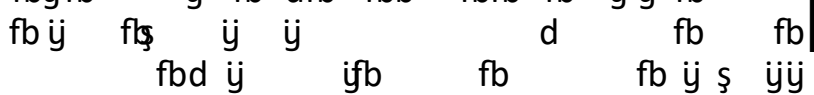

\subsection{The key actors in the region}

$\mathrm{J} \mathrm{fb}$ ij $\mathrm{fb} \ddot{\mathrm{y} f b} \dot{\mathrm{g}}$

$$
d \quad \text { ij } \quad \text { s }
$$

$\mathrm{fbd} \mathrm{ij}$

$\mathrm{fb} \dot{\mathrm{j}} \mathrm{ij} \mathrm{fb}$ ig

ij $\mathrm{fb} \dot{\mathrm{j}}$ $\mathrm{fbfb} f \mathrm{fb}$ $\mathrm{fb} \quad$ ij fbfb fb $\ddot{\mathrm{y}} \quad$ ij $\ddot{\mathrm{g}}$ fb ij fb ij fb Azerbaijan's fbfb fb $\ddot{\mathrm{y}} \dot{\mathrm{g}} \mathrm{ij} \quad \mathrm{fb}$ fbd $\mathrm{j}$ $\mathrm{fb} \quad \mathrm{fb} \quad \mathrm{fb} \quad \mathrm{fbş}$ idjijij ij $\dot{\text { is }}$ flfbij is

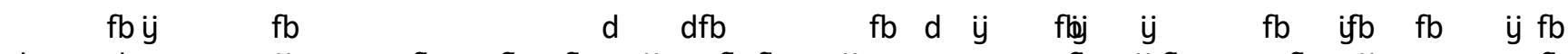

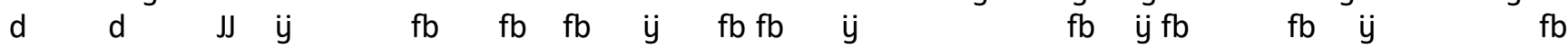

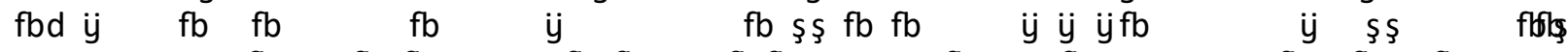

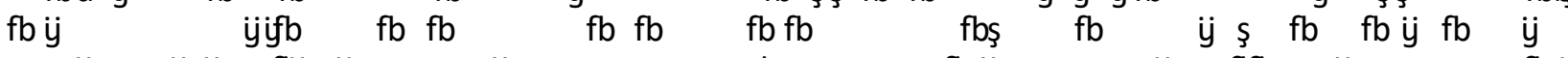

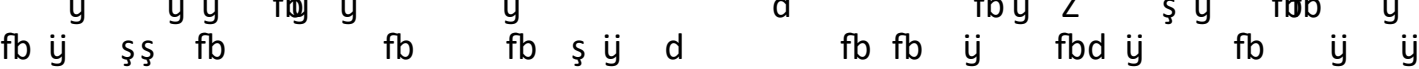
ifb

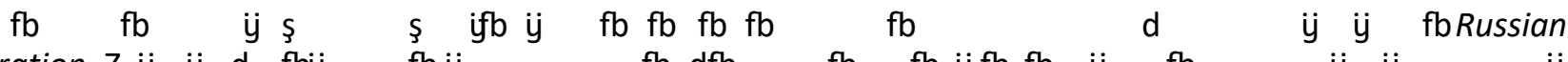

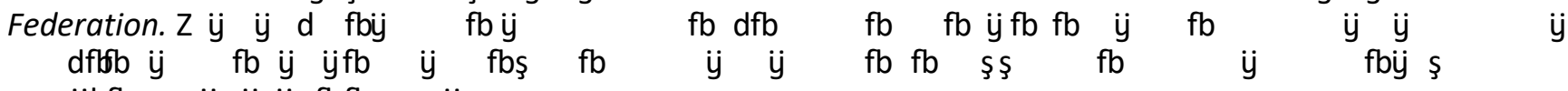

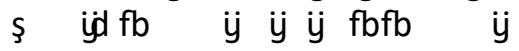
$J \quad \dot{y} \quad f b \quad f b \quad d \quad f b \ddot{y} \quad \ddot{y} \quad f b \quad f b i j \quad f b \quad \dot{y} \quad$ fbijş s $\quad \dot{y} \quad \ddot{y} \quad f b$

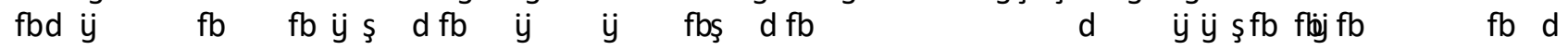

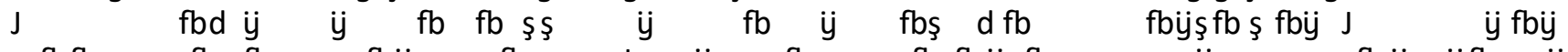

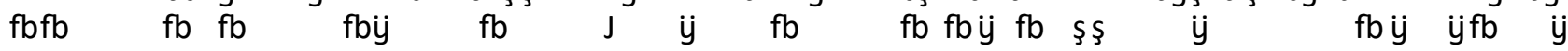

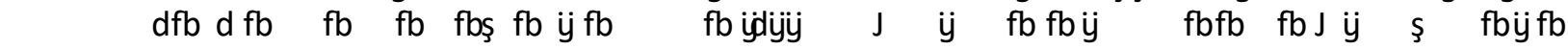
$J \quad \dot{y} d \ddot{y} f b \quad d \quad f b s \quad \dot{y}$

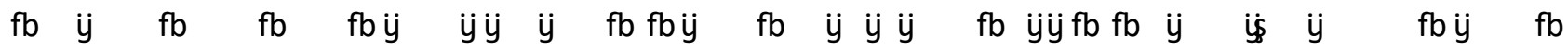

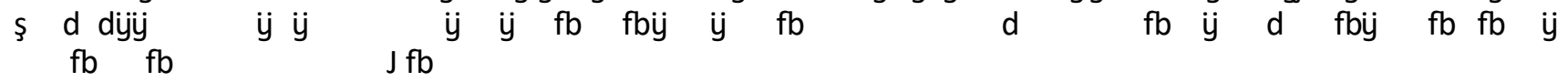


ij $\quad$ s

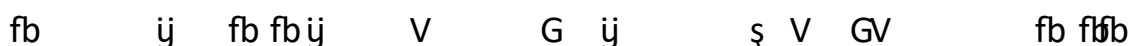
dfbş is $\dot{j}$ ij $\mathrm{fb} \quad \mathrm{fb} \quad \mathrm{fb} d \mathrm{fb} f \mathrm{fb}$ ij $\mathrm{fb}$

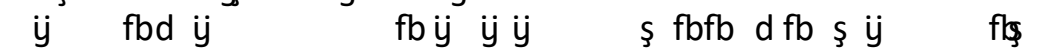
ij $\quad f b f b i j \quad$ ij $\quad f b$ ij fbş $d f b \quad f b f b$ ij $J$ ij $\ddot{y} \quad s \quad d d f b$ fbş $\dot{y} \quad$ ij $d f b$ fls $\mathrm{ijfb} \quad \mathrm{fb} f \mathrm{fb}$

$\mathrm{fb}$ flog $d f b$ ş $\ddot{j}$ ifb $\quad f b \quad d \quad f b i j f b$

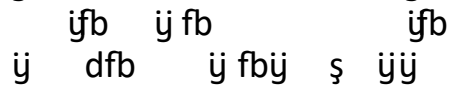
$\mathrm{fb} \quad \mathrm{j} \mathrm{dfb} d \mathrm{fb}$ ş $\mathrm{j}$

\subsection{Current developments}

$$
\begin{aligned}
& \text { ij } \mathrm{fb} \quad \mathrm{fb} f b \quad \mathrm{fb} \\
& \text { ij } \dot{y} \quad f b \\
& \mathrm{fb} \text { jïj } \\
& \mathrm{fb} \quad \mathrm{fb} f \mathrm{fb}
\end{aligned}
$$
J ij $\mathrm{fb} f b$

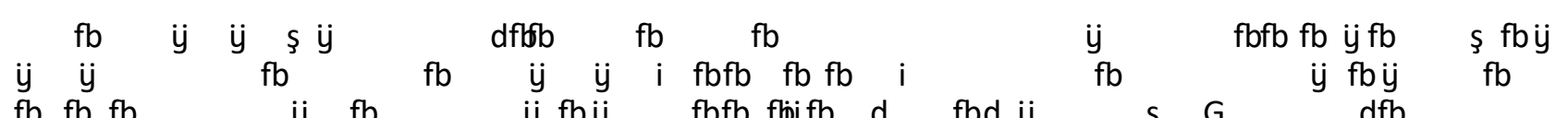

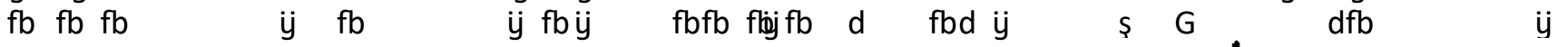

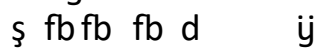

\section{Decision Analysis}

$f b \ddot{j} \dot{y} \quad$ i $\quad s \quad f b \quad \ddot{j} \quad s f b$ ij $\mathrm{fb} \ddot{\mathrm{y}} \mathrm{fb} \quad \mathrm{fb} \quad \mathrm{fb} \quad \mathrm{fb} \quad \mathrm{fb} \quad \mathrm{y} \quad \mathrm{fb}$ s $\ddot{j} f b \quad f b \quad$ ij $f b \quad$ fb $\quad$ fb $\ddot{y} \dot{y} \quad$ ij $\dot{y}$

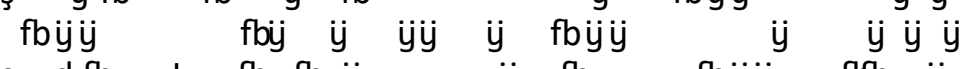

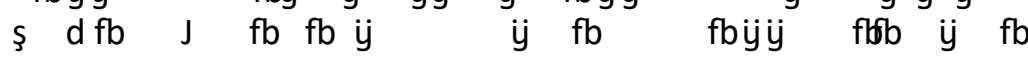
s $\mathrm{d} \mathrm{fb}$

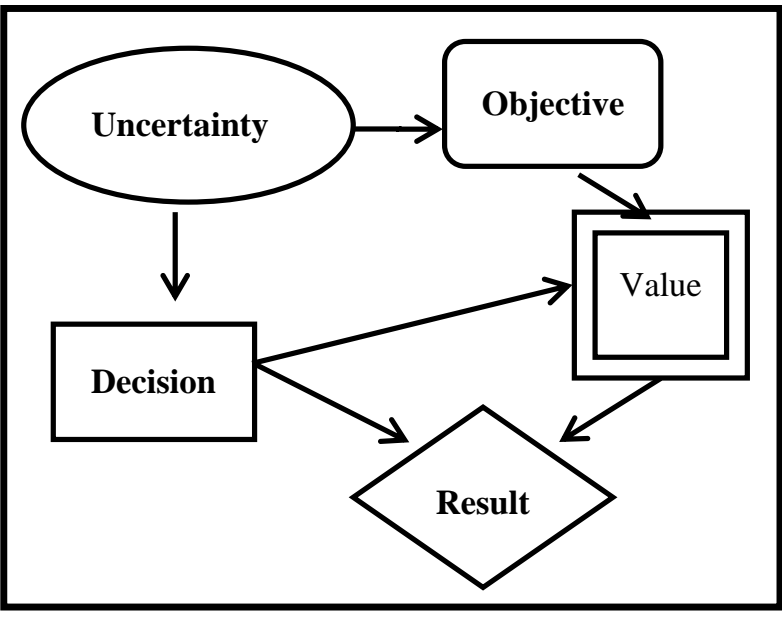

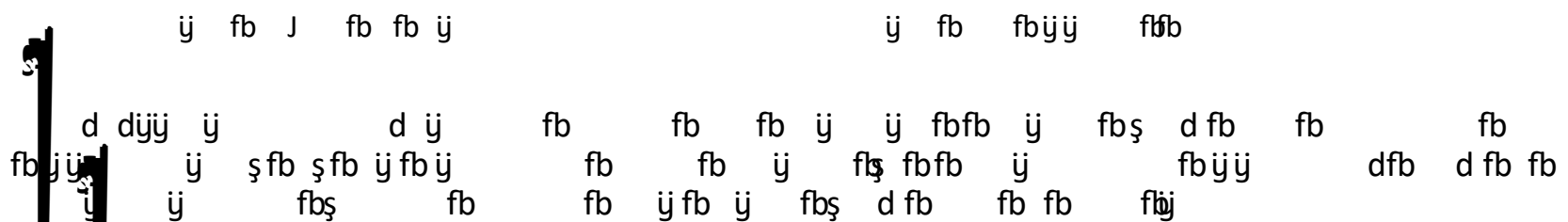

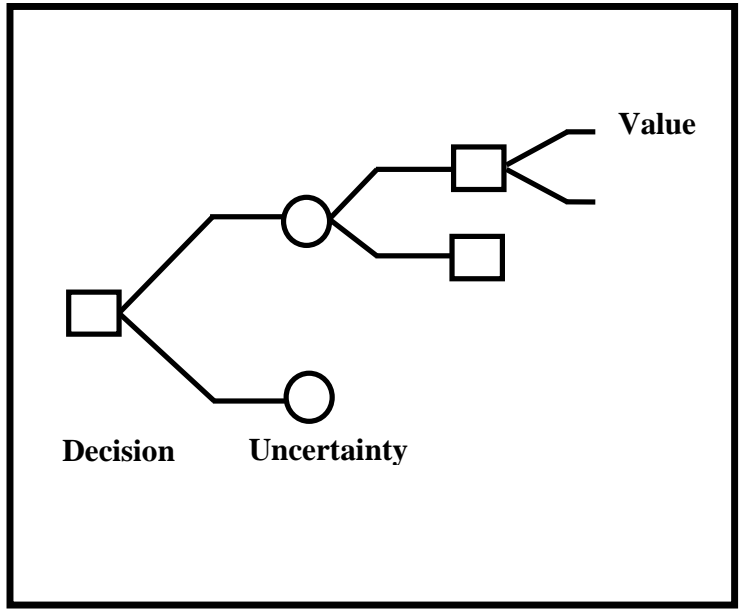

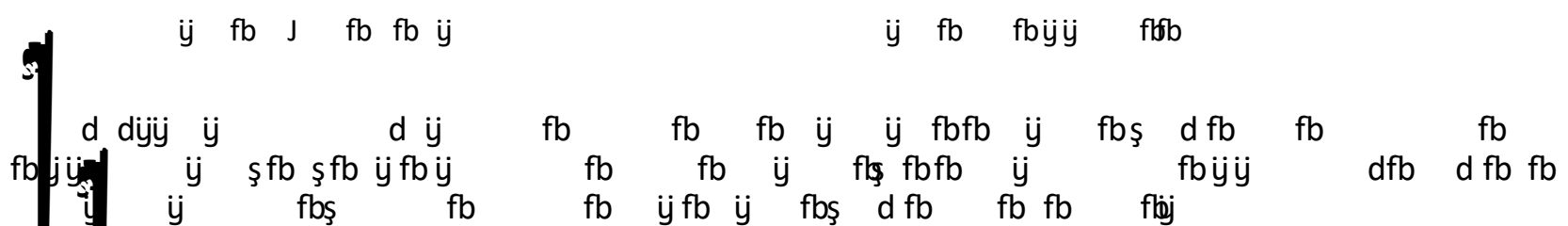

4. Mlod elling the Decision Problem

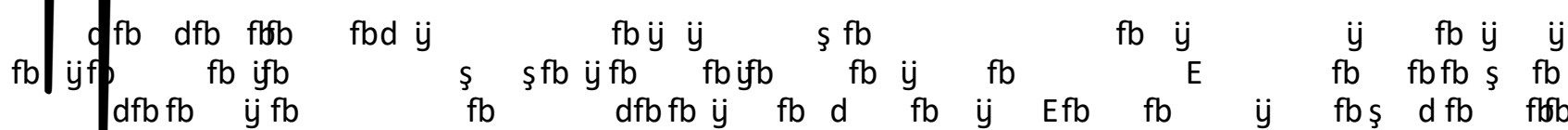

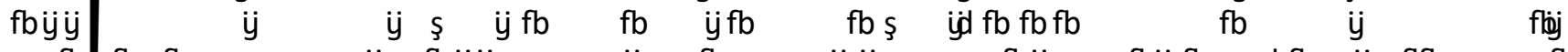

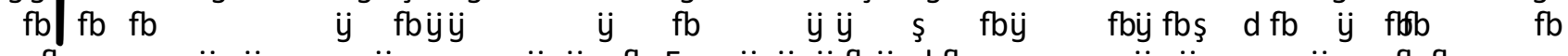

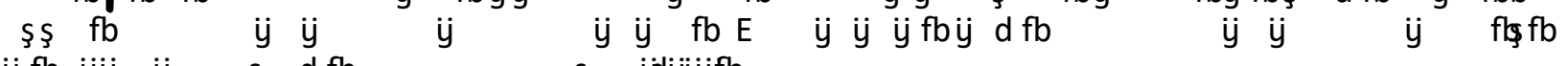

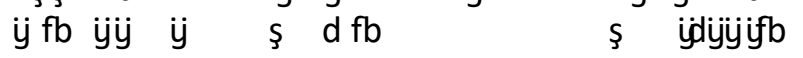




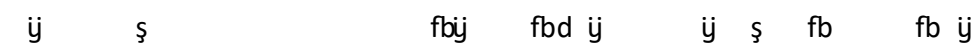

Journal of Sociology: Current Issues 7

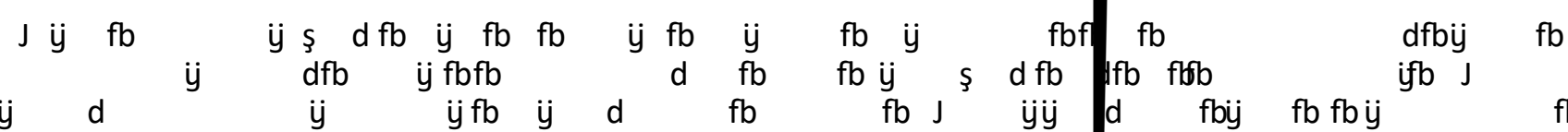

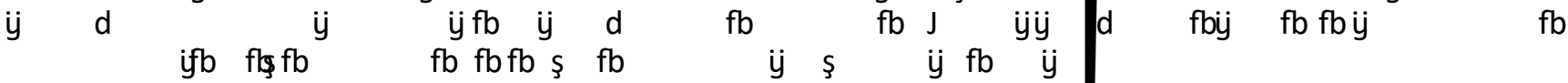

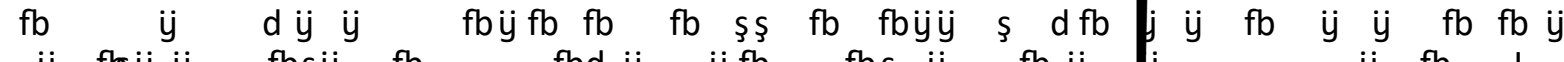

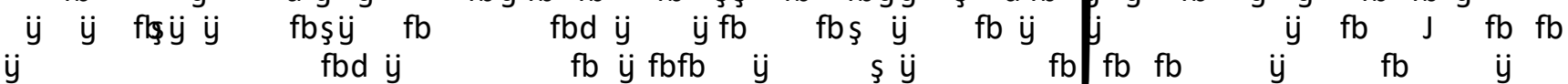
ij $\mathrm{flag} f \mathrm{fb}$ fbd $\dot{y} \quad d \quad \dot{y} \mathrm{fb} \quad \dot{y}$

$f$ flij $\mathrm{fb} \quad \mathrm{y} \quad \mathrm{i} \quad \mathrm{fb} \quad \mathrm{y}$ $\mathrm{fbd} \dot{\mathrm{y}} \quad \mathrm{dfb} \quad \mathrm{j} \mathrm{fb}$

- $\quad$ ij $\quad$ j

- $\quad$ ş flbb ij $\ddot{y}$ ifb

- $\quad J \mathrm{fb}$ ijfb is

- $\quad$ j $\quad \dot{y}$

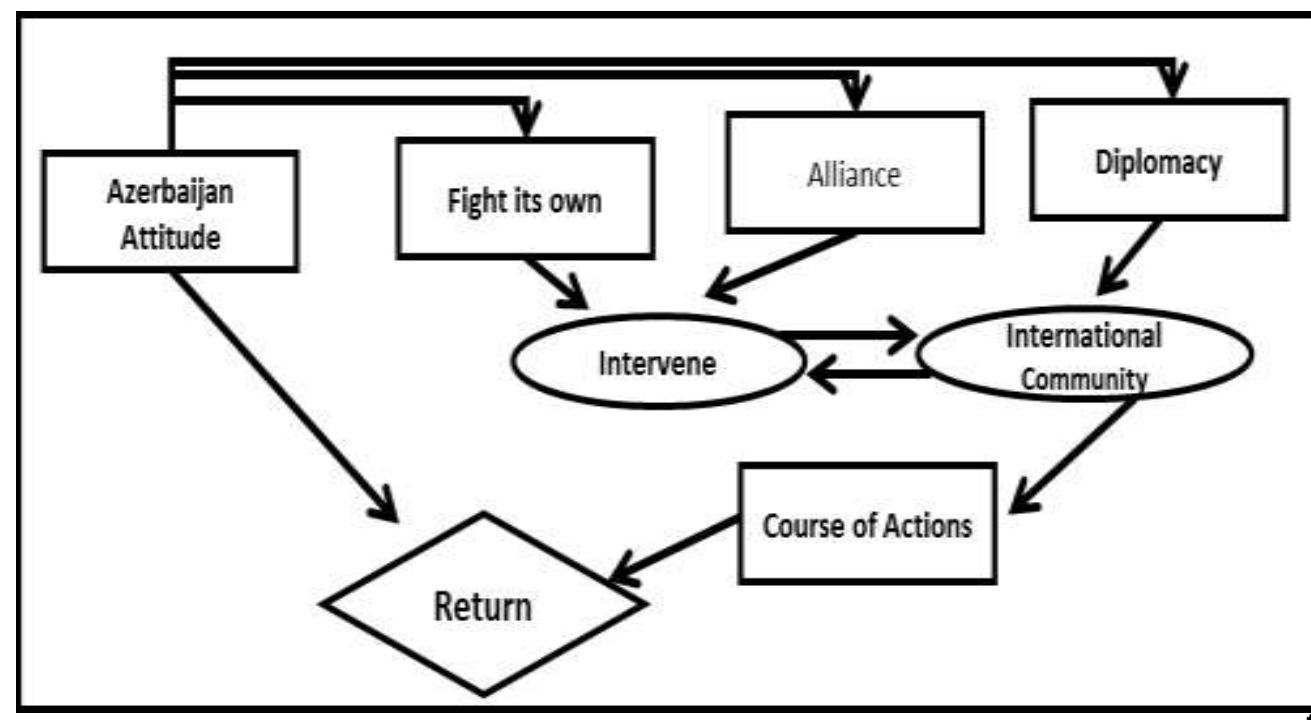

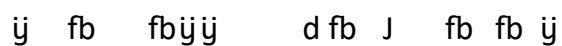

d $\quad$ şifb $\mathrm{fb}$ ij ifb

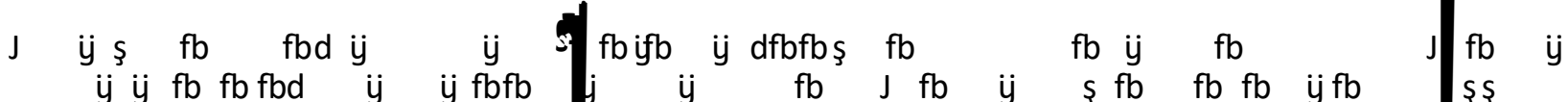

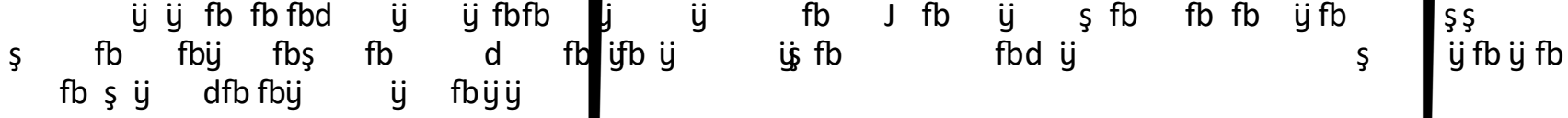

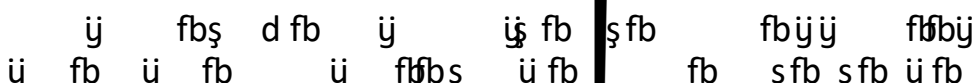
$\mathrm{fb}$ ij ifb

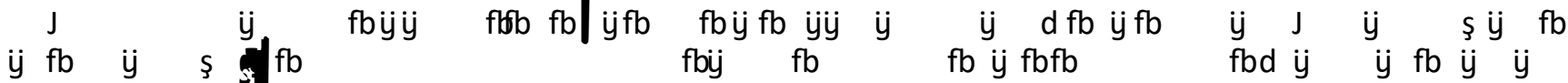

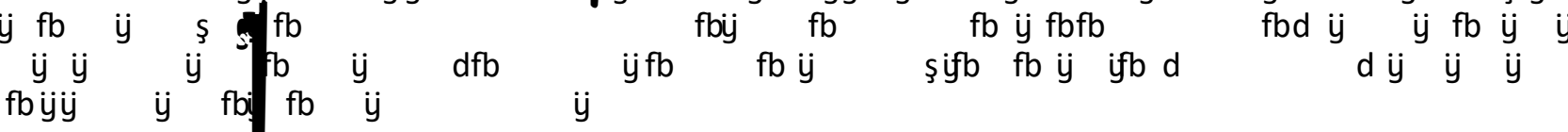

$\mathrm{fb} f b \ddot{y} \dot{y}$ flbb $\dot{y} \mathrm{fb}$ ij $\ddot{j} \mathrm{fb} f b$ ijs fbdfb flfb fbd $\dot{y}$

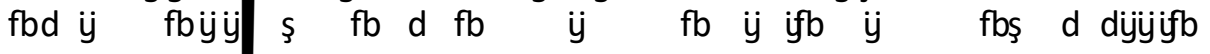

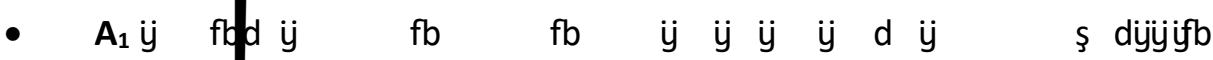

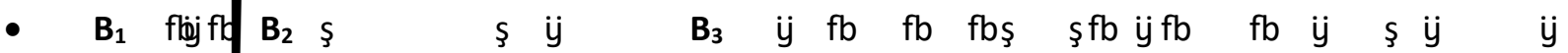
$\mathrm{fb} / \mathrm{fb} \quad \mathrm{fb} \quad \mathrm{y} \quad \mathrm{fb} \quad \mathrm{i}$

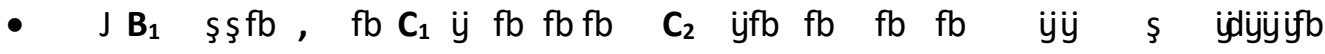

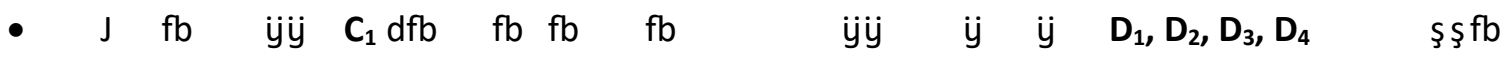



ij s fbij fbd

ij $\quad$ fb $\quad f b$ i

d

$\mathrm{fb} \quad$ şifb

\section{Conclusion}

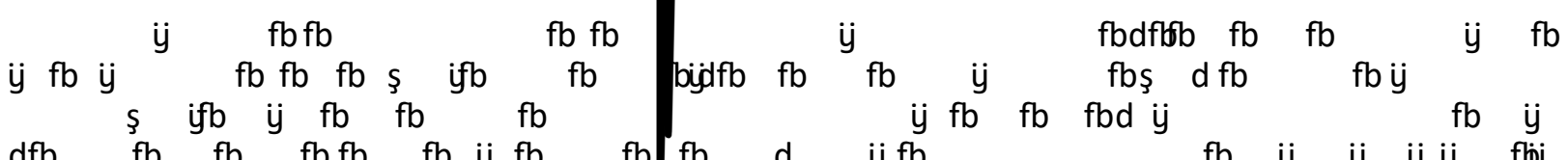

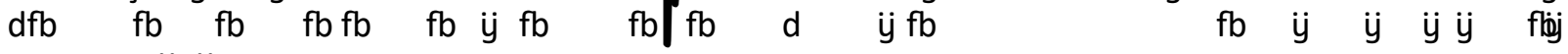
ij $\mathrm{y}$

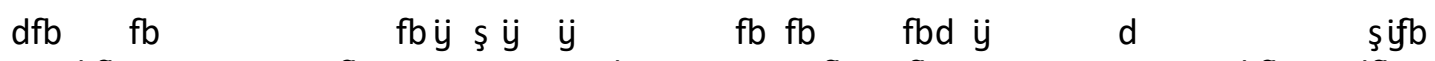

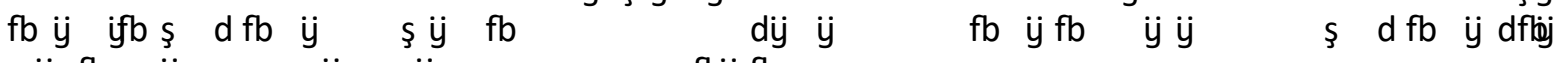

ij $\mathrm{fb}$ ij $\quad$ ij $\ddot{\mathrm{y}} \quad$ fbij fb

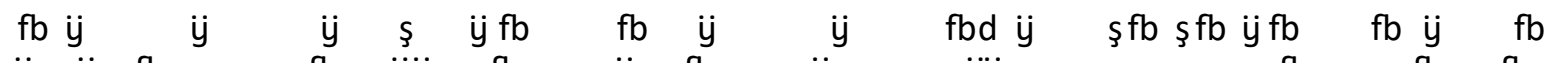

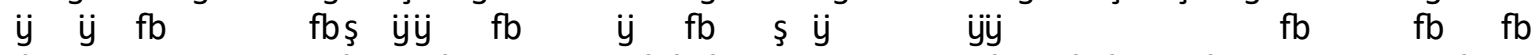
$\mathrm{fbfb} \mathrm{fb} \quad \mathrm{y} \quad$ s $\mathrm{fb} E$ fb $\mathrm{y} \quad \mathrm{fbfb} f \mathrm{fb}$ is fbş idiüigfb fbd $\ddot{y} \quad$ ij $\mathrm{fb} \quad \mathrm{fb} f \mathrm{~s} s \mathrm{fb} \quad$ ij fbij

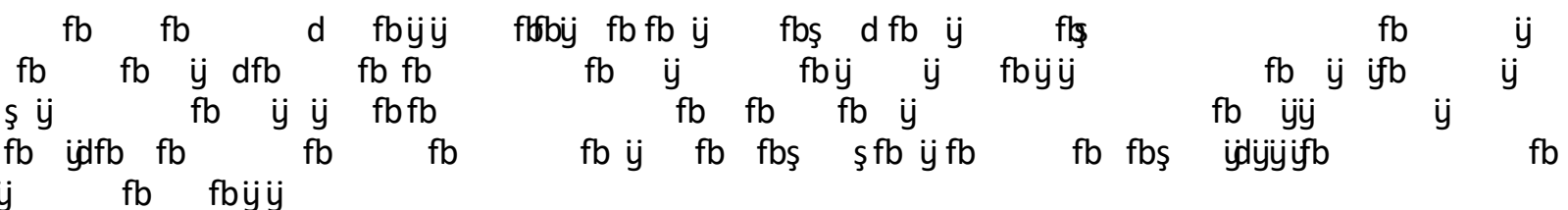

\section{References}

ij Armenian genocide. Armenian National Institute. $\mathrm{fb}$ ifb fb

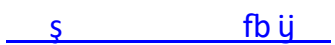
$\mathrm{fb} \quad \mathrm{i} f b \quad \mathrm{fb} \quad$ ij $f b$

ij $\quad$ ij $\mathrm{fb} f b \quad$ fb $\quad \mathrm{d} \quad$ Avrasya Dosyasi 7

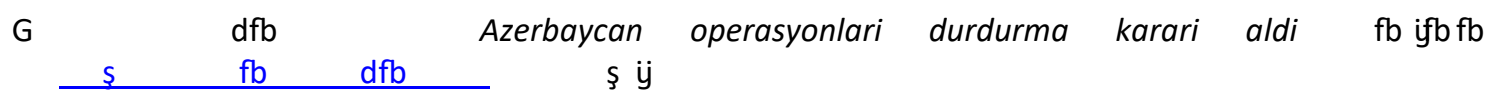

E $\quad$ g $\quad$ Karabag temel sorun. $\quad \dot{y} \dot{y} \mathrm{fb}$ fbfbig

E $\quad$ G fb

Turk Kulturu, 84

şij J $\mathrm{j} \quad \mathrm{fb} \mathrm{fb} \quad$ Azerbaijan and Armenia: Peace prospects, military realities \& the role of the armenian diaspora. $\mathrm{fb} \mathrm{ifb} \mathrm{fb}$ $\mathrm{fb}$ ij $\quad \mathrm{fbd}$ ij $\mathrm{fb} \mathrm{fb} \quad \mathrm{s} f \mathrm{~b}$

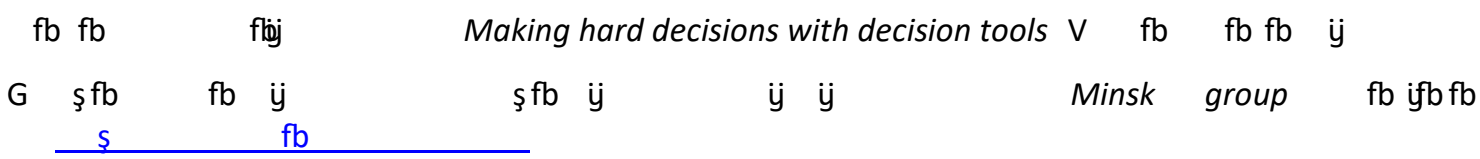
ij fbd $\quad$ ij fbij fb Yeni Turkiye Yay 19

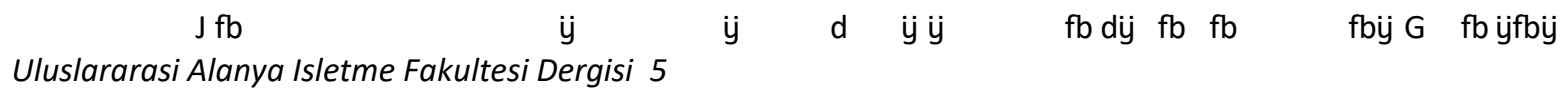

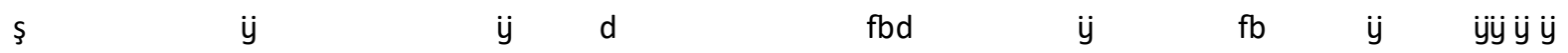
yj ì Guvenlik Stratejileri Dergisi 8

ij $E$

Istanbul, 200,

$\mathrm{dfb}$ fbfbij fb d Basbakanlik Osmanli Arsivi Yayini 

ig
ş
fbij fbd ij
ij ş fb
$\mathrm{fb} \dot{\mathrm{y}}$
d
$\mathrm{fb} \quad$ şfb

$\mathrm{fb}$ ig ifb Global Journal of Sociology: Current Issues 7

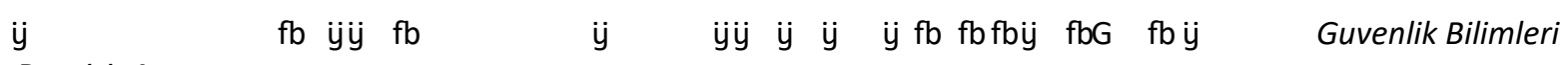

Dergisi 4

ì

d

Yeni Turkiye 3

ij $\ddot{y}$

$\mathrm{fb} \quad \mathrm{d} \quad$ ij $s \quad \mathrm{fb}$ Russia in Global Affairs, 2 